\title{
Calculation of critical curves of fluid mixtures through solution of differential equations \\ Mathematical Appendix
}

\author{
Ulrich K. Deiters \\ Institute of Physical Chemistry, University of Cologne, Greinstr. 4-6, 50939 Köln, Germany
}

Ian H. Bell

Applied Chemicals and Materials Div., National Institute of Science and Technology, Boulder, CO 80305, USA 


\section{S1. NUMERICAL DIFFERENTIATION}

The calculation of derivatives - often of higher order — of functions is a recurring task in thermodynamics. This can-and usually should—be done by analytical differentiation ("calculus"). But as most functions, with the exception of polynomials, tend to get more complicated upon differentiation, analytical differentiation is not always a practical option.

Of course analytical differentiation even of complicated functions can be performed by means of symbolicalgebra programs. Many of these, however, are expensive and not available to everybody. Furthermore, they may not be applicable if the function to be differentiated connot be written explicitly, for instance because it contains iterations.

In this context implicit analytic differentiation methods, like the autodiff software [1], should also be mentioned. The latter is restricted to $\mathrm{C}++$, however, at least at present.

It is therefore advantageous to consider numerical differentiation methods.

S1.1. Finite-Difference Methods. These methods assume that the function to be differentiated, $f(x)$, can be expanded into a Taylor series at the location $x$. Then the value of the function at $x+h$ is

$$
\begin{aligned}
f(x+h) & =f(x)+f^{\prime}(x) h+\frac{1}{2} f^{\prime \prime}(x) h^{2}+\frac{1}{6} f^{\prime \prime \prime}(x) h^{3}+\ldots \\
& =\sum_{k=0}^{\infty} \frac{1}{k !} f^{(k)}(x) h^{k}
\end{aligned}
$$

where the primes indicate the order of the derivative. When the difference of the function values at the locations $x+h$ and $x-h$ is calculated, the terms containing even powers of $h$ cancel:

$$
f(x+h)-f(x-h)=2 f^{\prime}(x) h+\frac{1}{3} f^{\prime \prime \prime}(x) h^{3}+\ldots .
$$

Rearrangement gives the first-order derivative,

$$
f^{\prime}(x)=\frac{1}{2 h}(f(x+h)-f(x-h))+O\left(h^{2}\right) .
$$

Adding instead of subtracting the function values at $x \pm h$ yields

$$
f(x+h)+f(x-h)=2 f(x)+f^{\prime \prime}(x) h^{2}+\ldots
$$

and, after rearrangement, a formula for the second-order derivative,

$$
f^{\prime \prime}(x)=\frac{1}{h^{2}}(f(x+h)-2 f(x)+f(x-h))+O\left(h^{2}\right) .
$$

These are already very useful results: The equations give good approximations for the first- and secondorder derivatives, and their errors decreases with the square of the size of the increment $h$. Unfortunately, however, it is not possible to make $h$ very small, for then the difference of the function values becomes unprecise because of the cancellation of digits. It is therefore necessary to weigh the cancellation error against the series truncation error $O\left(h^{2}\right)$. With "double precision" arithmetics, as it is presently defined by the standard IEEE 754, an increment size of $h \approx 10^{-5} x$ is usually a good compromise. 
An improvement can be achieved by computing additional function values and to use these to eliminate more terms of the Taylor series. For example, the difference of the function values at the locations $x \pm 2 h$ is

$$
f(x+2 h)-f(x-2 h)=4 f^{\prime}(x) h+\frac{8}{3} f^{\prime \prime \prime}(x) h^{3}+\ldots .
$$

Combining this equation with Eq. (S2) makes the cancellation of the third-order term possible,

$$
8(f(x+h)-f(x-h))-(f(x+2 h)-f(x-2 h))=12 f^{\prime}(x)+\ldots,
$$

which leads to

$$
f^{\prime}(x)=\frac{1}{12}(-f(x+2 h)+8 f(x+h)-8 f(x-h)+f(x-2 h))+O\left(h^{4}\right)
$$

as a better approximation.

Romberg proposed an iterative scheme for obtaining derivatives of arbitrary order [2]. The method requires the evaluation of the function at the locations $x \pm \alpha h, x \pm \alpha^{2} h$, etc., where $\alpha$ is an expansion factor usually chosen between 1.4 and 2 .

Ridders' method combines Romberg's scheme with a self-checking feature that terminates the iteration when no further reduction of the numerical uncertainty can be achieved [3].

An inherent disadvantage of all finite-difference methods for numerical differentiation is the cancellation of digits during the computation of the differences. The problem gets worse with increasing order of the differentiation. Romberg's or Ridders' methods alleviate the problem and can yield good results for firstand perhaps even second-order derivatives, but become increasingly unprecise for higher orders, unless the user employs a computing precision better than "double precision".

S1.2. Complex Integration The numerical problems of the finite-difference methods can be completely avoided by using methods based on complex-number algebra. Their basis is Cauchy's integral theorem, which relates the value of a function $f(z)$ or any of its to derivatives at the location $z_{0}$ to a path integral,

$$
\frac{\mathrm{d}^{n} f\left(z_{0}\right)}{\mathrm{d} z^{n}}=\frac{n !}{2 \pi} \oint_{C} \frac{f\left(z+z_{0}\right)}{z^{n}} \mathrm{~d} z
$$

where $C$ denotes a closed path in the complex plane that encloses $z_{0}$. The simplest choice is a circle with the radius $r$ centered at $z=z_{0}$. Then Cauchy's formula can be written as

$$
\frac{\mathrm{d}^{n} f\left(z_{0}\right)}{\mathrm{d} z^{n}}=\frac{n !}{2 \pi} \int_{0}^{2 \pi} \frac{f\left(z+z_{0}\right)}{z^{n}} \mathrm{~d} \phi \quad \text { with } \quad z \equiv r e^{i \phi} .
$$

The function $f(z)$ must evidently take a complex argument and return a complex value. But this does not constitute a practical problem, as modern computer languages like $\mathrm{C}++$ can handle complex algebra. Particular for $\mathrm{C}++$, a complex version of an existing subroutine for computing, e.g., the residual Helmholtz energy density $\Psi^{\mathrm{r}}(\vec{\rho}, T)$ can be provided by merely providing a template declaration.

The integration can be accomplished numerically by means of the trapezium rule. This is usually known as a rather inferior method, but for periodic functions it is very efficient: With increasing number of evaluation nodes $N$, the error of the integral decreases faster than any power of $2 \pi / N$.

The advantage of using an integral formula to obtain derivatives is that numerical integration-quite in contrast to numerical differentiation by means of finite-difference formulas-does not suffer from cancellation errors. If the radius of the integration path is chosen large enough (so large that $f\left(z+z_{0}\right)$ shows a significant variation), derivatives can be computed practically with machine precision. 
Even if we extend thermodynamic functions like the Helmholtz energy density $\Psi(\vec{\rho}, T)$ or $\Psi(\vec{\sigma}, T)$ to complex arguments and values, we must demand that these functions have real values and real derivatives when evaluated at real locations. For such functions the method of Lyness and Moler [4] is an interesting option. This method constructs a series of approximations $y_{m}$ for the $n$ th-order derivative,

$$
\begin{array}{r}
y_{m}=y_{m-1}+\frac{n !}{r^{n}} M_{m}\left[-\Re\left\{f\left(z_{0}\right)\right\}+\frac{1}{h} \sum_{k=0}^{m n-1} \Re\left\{f\left(z_{0}+r e^{2 \pi h k i}\right)\right\}\right], m=1,2, \ldots \\
\text { with } y_{0}=0 \text { and } h=\frac{1}{m n} .
\end{array}
$$

The $M_{m}$ are so-called Möbius numbers, which are defined as

$$
\begin{aligned}
M_{m} & = \begin{cases}+1 & \text { if } m=1 \text { or if } m \text { is a product of different prime numbers } \\
-1 & \text { if } m \text { is a prime number } \\
0 & \text { otherwise }\end{cases} \\
& =\{+1,-1,-1,0,-1,+1,-1,0,0,+1,-1,0,-1,+1,+1,0,-1,0,-1,0, \ldots\} .
\end{aligned}
$$

Convergence is usually quite rapid; the calculation can safely be terminated when the difference of two successive approximations falls below a predefined threshold.

S1.3. Finite Differences Using (Multi)Complex Algebra. Even when extended to complex algebra, the functions needed for this work have the property that they return real values for real arguments. For such functions, the algorithm of Squire and Trapp $[5,6]$ is a remarkably simple and efficient way to compute first-order derivatives:

$$
f^{\prime}(x)=\frac{1}{h} \Im\{f(x+h i)\} \quad \text { with } \quad h \approx 10^{-100}
$$

This is, in principle, a finite-difference method. But as the function argument is incremented along the imaginary axis and $\Im\{f(x)\}=0$ by definition, there is no loss of precision even if a very small increment is used.

It is advisable, however, to test this algorithm on the computer on which it is to be used, as it depends on the correct handling of limiting values by the complex-function library.

The method of Squire and Trapp can be extended to higher-order derivatives-including mixed derivatives-by using multicomplex algebra. Just as a complex number comprises two real numbers $(z=x+i y)$, a level-2 multicomplex number (also called bicomplex number) comprises two complex numbers,

$$
z^{(2)}=z_{0}^{(1)}+i^{(2)} z_{1}^{(1)}
$$

where the $z_{0,1}^{(1)}$ are complex numbers and $i^{(2)}$ is the imaginary unit of the second level. Similarly, a level-3 multicomplex number comprises two level-2 complex numbers and the imaginary unit of the third level, and so on. Complex numbers can be regarded as level-1 multicomplex numbers, and $i \equiv i^{(1)}$. Evidently, an $n$th level multicomplex number comprises $2^{n}$ real components.

An $n$th order derivative is then obtained from

$$
\frac{\mathrm{d}^{n} f(x)}{\mathrm{d} x^{n}}=\frac{1}{h^{n}} \Im^{n}\left\{f\left(x+i^{(1)} h+i^{(2)} h+\ldots+i^{(n)} h\right)\right\}
$$


where $\Im^{n}$ is an operator that returns the last, "most imaginary" real component of an $n$ th-level multicomplex number [7,8].

Without giving a formal proof we wish to point out that multicomplex differentiation can be used to obtain mixed derivatives of functions of more than one variable, e.g.,

$$
\left(\frac{\partial^{2} f(x, y)}{\partial x \partial y}\right)=\frac{1}{h^{2}} \Im^{2}\left\{f\left(x+i^{(1)} h, y+i^{(2)} h\right)\right\} .
$$

For the readers' convenience we have made Python and C++ libraries for multicomplex differentiation available [9].

\section{S2. DIRECTIONAL DERIVATIVES}

S2.1. Definitions. This article makes use of "directional derivatives", i.e. derivatives of thermodynamic functions-mostly the Helmholtz energy density - along a path specified by a direction vector. Let $f(\vec{x})$ denote a (scalar) function of a vector $\vec{x}=\left(x_{1}, \ldots, x_{N}\right)$, and $\vec{u}=\left(u_{1}, \ldots, u_{N}\right)$ a direction vector. Then the directional derivative of $f(\vec{x})$ is defined by [10]

$$
\frac{\partial f(\vec{x})}{\partial \vec{u}} \equiv \lim _{\xi \rightarrow 0} \frac{1}{\xi}(f(\vec{x}+\xi \vec{u})-f(\vec{x})) .
$$

$\vec{u}$ does not have to be a unit vector ${ }^{1}$; we merely demand that its length is not zero. $\xi$ is a scalar diplacement parameter.

The total differential of $f$ is

$$
\mathrm{d} f=\sum_{i=1}^{N}\left(\frac{\partial f}{\partial x_{i}}\right)_{x_{j \neq i}} \mathrm{~d} x_{i} .
$$

If we now set

$$
\mathrm{d} x=\vec{u} \mathrm{~d} \xi
$$

the total differential of $f$ becomes

$$
\mathrm{d} f=\sum_{i=1}^{N}\left(\frac{\partial f}{\partial x_{i}}\right)_{x_{j \neq i}} u_{i} \mathrm{~d} \xi,
$$

and then division by $\mathrm{d} \xi$ yields the directional derivative

$$
\frac{\partial f}{\partial \vec{u}}=\sum_{i=1}^{N}\left(\frac{\partial f}{\partial x_{i}}\right)_{x_{j \neq i}} u_{i}=\vec{u} \cdot \nabla f .
$$

\footnotetext{
${ }^{1}$ The mathematical literature is not uniform about this.
} 
If $\vec{u}$ is a unit vector aligned with one of the coordinate axes, the directional derivative coincides with the associated partial derivative,

$$
\vec{u}=\left(\begin{array}{c}
0 \\
\vdots \\
u_{k}=1 \\
\vdots \\
0
\end{array}\right) \Rightarrow \frac{\partial f}{\partial \vec{u}}=\left(\frac{\partial f}{\partial x_{k}}\right)_{x_{i \neq k}}
$$

and an all-1 vector yields

$$
\vec{u}=\left(\begin{array}{c}
1 \\
\vdots \\
1
\end{array}\right) \Rightarrow \frac{\partial f}{\partial \vec{u}}=\sum_{i=1}^{N}\left(\frac{\partial f}{\partial x_{i}}\right)_{x_{j \neq i}}
$$

In the particular case that there is an complete orthonormal set of direction vectors $\vec{u}_{k}, k=1, \ldots, N$, so that a variation along one vector does not imply a variation along any of the others, the directional derivatives can be regarded as partial derivatives of the $\vec{u}$ space,

$$
\frac{\partial f(\vec{x})}{\partial \vec{u}_{k}}=\left(\frac{\partial f(\vec{\xi})}{\partial \xi_{k}}\right)_{\xi_{i \neq k}}, k=1, \ldots, N .
$$

The $\xi_{i}$ are coordinates of the $\vec{u}$ space.

S2.2. Directional Derivatives of $\Psi$. According to Eq. (S5), the directional derivative along an arbitrary direction vector $\vec{u}$ at constant temperature, using a concentration displacement $\mathrm{d} \vec{\rho}=\vec{u} \mathrm{~d} \sigma$, is

$$
\frac{\partial \Psi}{\partial \vec{u}}=\sum_{i=1}^{N} \mu_{i} u_{i}=\vec{\mu} \cdot \vec{u} .
$$

If $\vec{u}$ is a unit vector of the $\vec{\rho}$ space, the directional derivative is the associated chemical potential (cf. Eq. (S6)),

$$
\vec{u}=\left(\begin{array}{c}
0 \\
\vdots \\
u_{k}=1 \\
\vdots \\
0
\end{array}\right) \Rightarrow \frac{\partial \Psi}{\partial \vec{u}}=\mu_{k}
$$

According to Eq. (S7), setting all elements of $\vec{u}$ to 1 gives

$$
\vec{u}=\left(\begin{array}{c}
1 \\
\vdots \\
1
\end{array}\right) \Rightarrow \frac{\partial \Psi}{\partial \vec{u}}=\sum_{i=1}^{N} \mu_{i}=p+\Psi
$$


which provides a useful way to obtain the pressure from $\Psi$, which requires merely one differentiation instead of $N$ as in Eq. (11) of the article.

We now use the $k$ th eigenvector of the Hessian matrix $\boldsymbol{\Psi}$ as directional vector, remembering at the same time that the Helmholtz energy density $\Psi$ of a fluid mixture can be written as the sum of an intrinsic part (which contains thermochemical data which are not relevant for this work), an ideal-mixing term, and a residual term,

$$
\Psi=\Psi^{\ominus}+R T \sum_{i=1}^{N} \rho_{i} \ln \rho_{i}+\Psi^{\mathrm{r}}
$$

Then Eq. (S9) becomes

$$
\frac{\partial \Psi}{\partial \vec{u}_{k}}=\vec{\mu}^{\mathrm{r}} \cdot \vec{u}_{k}+R T \sum_{i=1}^{N}\left(\ln \rho_{i}+1\right) u_{k, i} .
$$

As the eigenvectors $\vec{u}_{k}$ form an orthonormal basis, the path derivative can be regarded as a partial derivative with respect to $\sigma_{k}$, the displacement in $\vec{u}_{k}$-direction, and we may write

$$
\left(\frac{\partial \Psi}{\partial \sigma_{k}}\right)_{\sigma_{j \neq k}}=\frac{\partial \Psi}{\partial \vec{u}_{k}}=\vec{\mu}^{\mathrm{r}} \cdot \vec{u}_{k}+R T \sum_{i=1}^{N}\left(\ln \rho_{i}+1\right) u_{k, i} .
$$

Next we form the total differential of this derivative,

$$
\mathrm{d}\left(\frac{\partial \Psi}{\partial \sigma_{k}}\right)=\sum_{i=1}^{N}\left(\frac{\partial^{2} \Psi}{\partial \sigma_{k} \partial \rho_{i}}\right) \mathrm{d} \rho_{i}=\sum_{i=1}^{N}\left(\frac{\partial^{2} \Psi}{\partial \sigma_{k} \partial \rho_{i}}\right) u_{k, i} \mathrm{~d} \sigma_{k} .
$$

After division by $\mathrm{d} \sigma_{k}$ as well as splitting $\Psi$ into ideal and residual parts we obtain the second-order directional derivative,

$$
\left(\frac{\partial^{2} \Psi}{\partial \sigma_{k}^{2}}\right)=\frac{\partial\left(\partial \Psi / \partial \vec{u}_{k}\right)}{\partial \vec{u}_{k}}=\sum_{i=1}^{N} \sum_{j=1}^{N}\left(\frac{\partial \mu_{j}}{\partial \rho_{i}}\right) u_{k, j}=\underbrace{\sum_{i=1}^{N} \sum_{j=1}^{N}\left(\frac{\partial \mu_{j}^{\mathrm{r}}}{\partial \rho_{i}}\right) u_{k, j}}_{\left(\partial^{2} \Psi^{\mathrm{r}} / \partial \sigma_{k}^{2}\right)}+R T \sum_{i=1}^{N} \frac{u_{k, i}^{2}}{\rho_{i}}
$$

Repeating the directional differentiation along $\vec{u}_{k}$ gives expressions for the third- and fourth-order derivatives,

$$
\left(\frac{\partial^{3} \Psi}{\partial \sigma_{k}^{3}}\right)=\left(\frac{\partial^{3} \Psi^{\mathrm{r}}}{\partial \sigma_{k}^{3}}\right)-R T \sum_{i=1}^{N} \frac{u_{k, i}^{3}}{\rho_{i}^{2}}
$$

and

$$
\left(\frac{\partial^{4} \Psi}{\partial \sigma_{k}^{4}}\right)=\left(\frac{\partial^{4} \Psi^{\mathrm{r}}}{\partial \sigma_{k}^{4}}\right)+R T \sum_{i=1}^{N} \frac{2 u_{k, i}^{4}}{\rho_{i}^{3}}
$$


S2.3. Mixed Derivatives. Directional derivatives can used to obtain mixed higher-order derivatives from pure derivatives. Forming the total differential of Eq. (S5) yields

$$
\mathrm{d} \frac{\partial f}{\partial \vec{u}}=\sum_{j=1}^{N} \frac{\partial}{\partial x_{j}}\left(\sum_{i=1}^{N}\left(\frac{\partial f}{\partial x_{i}}\right) u_{i}\right) \mathrm{d} x_{j} .
$$

Using Eq. (S3) again and dividing by $\mathrm{d} \xi$ then gives

$$
\frac{\partial^{2} f}{\partial \vec{u}^{2}}=\sum_{j=1}^{N} \sum_{i=1}^{N}\left(\frac{\partial^{2} f}{\partial x_{i} \partial x_{j}}\right) u_{i} u_{j} .
$$

For simplicity we now assume $N=2$, but the following equations can easily be generalized to arbitary values of $N$. We can then set

$$
\begin{aligned}
& \vec{u}=\left(\begin{array}{l}
1 \\
0
\end{array}\right) \quad \Rightarrow \quad f_{10}=\frac{\partial f}{\partial \vec{u}}=\left(\frac{\partial^{2} f}{\partial x_{1}^{2}}\right) \\
& \vec{u}=\left(\begin{array}{l}
0 \\
1
\end{array}\right) \Rightarrow f_{01}=\frac{\partial f}{\partial \vec{u}}=\left(\frac{\partial^{2} f}{\partial x_{2}^{2}}\right) \\
& \vec{u}=\left(\begin{array}{l}
1 \\
1
\end{array}\right) \Rightarrow f_{11}=\frac{\partial f}{\partial \vec{u}}=\left(\frac{\partial^{2} f}{\partial x_{1}^{2}}\right)+2\left(\frac{\partial^{2} f}{\partial x_{1} \partial x_{2}}\right)+\left(\frac{\partial^{2} f}{\partial x_{2}^{2}}\right) .
\end{aligned}
$$

Consequently

$$
\left(\frac{\partial^{2} f}{\partial x_{1} \partial x_{2}}\right)=\frac{1}{2}\left(f_{11}-f_{10}-f_{01}\right)
$$

is an expression for the mixed derivative.

It should be noted that Eq. (S22) is not a finite-difference formula like the equations in Appendix 1.1 and therefore does not necessarily suffer from cancellation errors.

This way to calculate mixed derivatives is advantageous if complex integration methods are used (Appendix 1.2), for the generalization of such methods to multidimensional functions is rather complicated.

\section{SYMBOLS}

$h \quad$ increment in numerical differentiation

$i^{(k)} \quad$ imaginary unit of the $k$ th level

$\Im \quad$ imaginary part of a (multi)complex number

$N \quad$ number of components of a mixture

$p \quad$ pressure

$R \quad$ gas constant

$\Re \quad$ real part of a (multi)complex number

$T$ temperature

$\vec{u}_{k} \quad k$ th eigenvector, $\vec{u}_{k}=\left(u_{k, 1}, \ldots, u_{k, N}\right)$ 
$z^{(k)} \quad$ multicomplex number, level $k$

$\vec{\mu} \quad$ vector of chemical potentials, $\vec{\mu}=\left(\mu_{1}, \ldots, \mu_{N}\right)$

$\vec{\rho} \quad$ vector of molar concentrations, $\vec{\rho}=\left(\rho_{1}, \ldots, \rho_{N}\right)$

$\vec{\sigma} \quad$ vector of transformed concentrations, $\vec{\sigma}=\left(\sigma_{1}, \ldots, \sigma_{N}\right)$

$\Psi \quad$ Helmholtz energy density

$\boldsymbol{\Psi} \quad$ Hessian matrix of $\Psi, \boldsymbol{\Psi}=\left(\left\{\Psi_{i j}\right\}\right), \Psi_{i j}=\left(\partial^{2} \Psi / \partial \rho_{i} \partial \rho_{j}\right)$

\section{Sub- and Superscripts}

r residual property

$\theta \quad$ reference state

\section{REFERENCES}

(1) Leal, A. autodiff (automatic differentiation for C++). https://github.com/autodiff/autodiff, 2018-2019.

(2) Jordan-Engeln, G.; Reutter, F. Numerische Mathematik für Ingenieure. Bibliographisches Institut, Mannheim, 1985.

(3) Press, W. H.; Teukolsky, S. A.; Vetterling, W. T.; Flannery, B. P. Numerical Recipes in C. Cambridge University Press, Cambridge, 2002.

(4) Lyness, J. N.; Moler, C. B. Numerical differentiation of analytic functions. SIAM J. Numer. Anal. 1967, 4, 202-210.

(5) Squire, W.; Trapp, G. Using complex variables to estimate derivatives of real functions. SIAM Rev. 1998, 40, 110-112.

(6) Higham, N. Differentiation with(out) a difference. SIAM News 2018, 51(5), 2.

(7) Lantoine, G.; Russell, R. P.; Dargent, Th. Using multicomplex variables for automatic computation of high-order derivatives. ACM Trans. Math. Softw. 2012, 38, no. 16.

(8) Verheyleweghen, A. Computation of higher-order derivatives using the multi-complex step method. Technical report, NTNU, 2014.

(9) Bell, I. H. mcx (multicomplex algebra library). https://github.com/ianhbell/mcx, 2019.

(10) https://en.wikipedia.org/wiki/Directional_derivative. accessed on 2018-02-21. 